ICRR-Report-317-94-12

April 1994

\title{
Flavor mixing in the gluino coupling and the nucleon decay
}

\author{
Toru Goto, Takeshi Nihei and Jiro Arafune \\ Institute for Cosmic Ray Research, University of Tokyo, \\ Midori-cho, Tanashi-shi, Tokyo 188 JAPAN
}

\begin{abstract}
Flavor mixing in the quark-squark-gluino coupling is studied for the minimal SU(5) SUGRA-GUT model and applied to evaluation of the nucleon lifetime. All off-diagonal (generation mixing) elements of Yukawa coupling matrices and of squark/slepton mass matrices are included in solving numerically one-loop renormalization group equations for MSSM parameters, and the parameter region consistent with the radiative electroweak symmetry breaking condition is searched. It is shown that the flavor mixing in the gluino coupling for a large $\tan \beta$ is of the same order of magnitude as the corresponding Kobayashi-Maskawa matrix element in both up-type and down-type sector. There exist parameter regions where the nucleon decay amplitudes for charged lepton modes are dominated by the gluino dressing process, while for all the examined regions the neutrino mode amplitudes are dominated by the wino dressing over the gluino dressing.
\end{abstract}




\section{Introduction}

$\mathrm{SU}(5)$ supersymmetric (SUSY) grand unified theory (GUT) is an attractive candidate for the unified theory of strong and electroweak interactions. The analyses of the gauge coupling unification [1] suggest the validity of the minimal supersymmetric standard model (MSSM) just above the electroweak scale $\sim 100 \mathrm{GeV}$ and the unification of $\mathrm{SU}(3) \times \mathrm{SU}(2) \times \mathrm{U}(1)$ gauge group into a simple $\mathrm{SU}(5)$ at the $\mathrm{GUT}$ scale $M_{X} \sim 10^{16} \mathrm{GeV}$.

One of the features of MSSM/SUSY-GUT is the existence of soft SUSY breaking. It gives quarks (or leptons) and their superpartners different mass matrices in the generation (flavor) space. In results, due to the discrepancy between the mass diagonalizing bases of quarks and those of squarks, a generation mixing occurs in the quark-squark-gaugino coupling (gluino coupling, in particular), which may give considerable contributions to the nucleon decay, flavor changing neutral currents (FCNC) and other various phenomena in SUSY-GUT. The flavor mixing in the gaugino coupling plays an important role in the nucleon decay, since its amplitude is dominated by the dimension five interaction followed by the gaugino "dressing" process [2] in the minimal SU(5) SUSY-GUT model. However, only a simplified

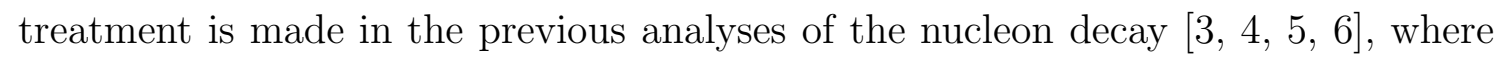
the diagonal (in the generation space) gluino coupling is assumed leading to the negligible contribution from the gluino dressing process.

The purpose of the present paper is to study the flavor mixing in the gaugino coupling extensively and evaluate the contribution of the gluino dressing process to the nucleon decay amplitude. Based on the minimal SU(5) supergravity (SUGRA-) GUT [7], we assume that the soft SUSY breaking parameters are "universal" at the GUT scale [8]. We include all off-diagonal elements of Yukawa coupling matrices and of squark/slepton mass matrices in solving numerically one-loop renormalization group equations (RGEs) for all MSSM parameters [10, 11] with the universal boundary conditions. We then evaluate the effective potential for the Higgs fields at the electroweak scale to find a consistent $\mathrm{SU}(2) \times \mathrm{U}(1)$ breaking minimum in accordance with the radiative electroweak symmetry breaking scenario [10]. The obtained mass matrices of all particles are diagonalized to evaluate the flavor mixing in the

\footnotetext{
${ }^{*}$ Two-loop RGEs for soft SUSY breaking parameters are obtained recently [9], which will be important for more accurate analysis.
} 
gaugino couplings. The mass spectrum and the mixing are then used to calculate the nucleon decay amplitudes for various decay modes. It is found that the flavor mixing in the gluino coupling depends on $\tan \beta$ (ratio of vacuum expectation values of the Higgs doublets) and is roughly of the same order of magnitude as the corresponding Kobayashi-Maskawa matrix element. As for the nucleon decay, we find the gluino dressing process dominates the amplitude for the decay modes containing a charged lepton (and a meson) if $\tan \beta$ is large and if the gluino mass is much smaller than the squark masses. Note that the flavor mixing in the gaugino couplings is studied previously in a systematic analysis of FCNC [11] with a semi-analytic solution of the RGEs for small tan $\beta$ (top Yukawa coupling $\gg$ bottom Yukawa coupling). On the contrary, our numerical method cover the whole range of $\tan \beta$, since all Yukawa couplings are taken into account.

The remaining part of this paper is organized as follows. After introducing in the next section the minimal SU(5) SUGRA-GUT model which we consider, we formulate the flavor mixing in the gaugino couplings in Sec. III. In Sec. IV the nucleon decay amplitudes are obtained with a careful treatment of the flavor mixing. The outline of the numerical calculation and the results are presented in Sec. $\nabla$ and our conclusions are summarized in Sec. V1.

\section{Minimal SU(5) SUGRA-GUT model}

Minimal SU(5) SUSY-GUT model contains three generations of matter multiplets with 10 and $\overline{\mathbf{5}}$ representations, $\Psi_{i}^{A B}$ and $\Phi_{j A}$ respectively, where suffices $A, B=$ $1,2, \cdots, 5$ are $\mathrm{SU}(5)$ indices and $i, j=1,2,3$ are the generation labels, and three kinds of Higgs multiplets with 5, $\overline{5}$ and 24 representations, $H_{5}^{A}, H_{\overline{5} A}$ and $\Sigma_{B}^{A}$ respectively. $\mathrm{SU}(5)$ and R-parity invariant superpotential $W_{\mathrm{GUT}}$ at the GUT scale is written as

$$
\begin{aligned}
W_{\mathrm{GUT}}\left(M_{X}\right)= & f^{i j} \Psi_{i}^{A B} \Phi_{j A} H_{\overline{5} B}+\frac{1}{8} g^{i j} \epsilon_{A B C D E} \Psi_{i}^{A B} \Psi_{j}^{C D} H_{5}^{E} \\
& +\lambda H_{\overline{5} A}\left(\Sigma_{B}^{A}+M \delta_{B}^{A}\right) H_{5}^{B}+W_{\Sigma}(\Sigma)
\end{aligned}
$$

Here, $\epsilon_{A B C D E}$ is the totally antisymmetric constant, $f^{i j}, g^{i j}=g^{j i}$ and $\lambda$ are dimensionless couplings, $M$ is a mass parameter and $W_{\Sigma}$ is a self-interaction superpotential for $\Sigma_{B}^{A}$. SU(5) symmetry is spontaneously broken down to $\mathrm{SU}(3) \times \mathrm{SU}(2) \times \mathrm{U}(1)$ 
with the nonvanishing vacuum expectation value of the adjoint Higgs $\Sigma_{B}^{A}$. Below the GUT scale, the model is reduced to MSSM with effective higher dimensional operators, which are obtained by integrating out the superheavy particles in Eq. (2.1). The effective superpotential is then written as

$$
\begin{aligned}
W_{\mathrm{eff}}= & W_{\mathrm{MSSM}}+W_{5}+O\left(M_{X}^{-2}\right), \\
W_{\mathrm{MSSM}}= & f_{D}^{i j} Q_{i}^{a \alpha} D_{j a} H_{1 \alpha}+f_{L}^{i j} \epsilon^{\alpha \beta} E_{i} L_{j \alpha} H_{1 \beta}+g_{U}^{i j} \epsilon_{\alpha \beta} Q_{i}^{a \alpha} U_{j a} H_{2}^{\beta}+\mu H_{1 \alpha} H_{2}^{\alpha}, \\
W_{5}= & \frac{1}{M_{C}}\left\{\frac{1}{2} C_{L}^{i j k l} \epsilon_{a b c} \epsilon_{\alpha \beta} Q_{k}^{a \alpha} Q_{l}^{b \beta} Q_{i}^{c \gamma} L_{j \gamma}+C_{R}^{i j k l} \epsilon^{a b c} U_{i a} D_{j c} E_{k} U_{l b}\right. \\
& \quad+\text { (baryon number/lepton number conserving terms) }\} .
\end{aligned}
$$

Here, $Q, U$ and $E$ are chiral superfields which contain left-handed quark doublet, right-handed up-type quark and right-handed charged lepton respectively, and are embedded in $\Psi$ (to be specified in Eq. (3.4)); $D$ and $L$, which are embedded in $\Phi$, contain right-handed down-type quark and left-handed lepton doublet respectively; $H_{1}$ and $H_{2}$ are Higgs doublets embedded in $H_{\overline{5}}$ and $H_{5}$, respectively. The suffices $a, b, c=1,2,3$ are $\mathrm{SU}(3)$ indices and $\alpha, \beta=1,2$ are $\mathrm{SU}(2)$ indices. $M_{C}$ is the colored Higgs mass which is assumed to be $O\left(M_{X}\right)$, while the supersymmetric mass of Higgs doublet $\mu$ is of the order of the $Z$ boson mass $m_{Z}$. This discrepancy is owing to a tree level fine-tuning in the GUT superpotential. At the GUT scale, $f_{D}$ and $f_{L}$ are unified and $C_{L}$ and $C_{R}$ are written in terms of the Yukawa coupling constants (see Sec. [V). Baryon number (and lepton number) violating terms in $W_{5}$ give dominant contributions to the nucleon decay in this model.

In addition to the supersymmetric Lagrangian to be derived from (2.2), the following soft SUSY breaking terms are included:

$$
\begin{aligned}
-\mathcal{L}_{\text {soft }}= & \left(m_{Q}^{2}\right)_{i}^{j} \tilde{q}^{\dagger i} \tilde{q}_{j}+\left(m_{D}^{2}\right)_{i}^{j} \tilde{d}^{\dagger i} \tilde{d}_{j}+\left(m_{U}^{2}\right)_{i}^{j} \tilde{u}^{\dagger i} \tilde{u}_{j} \\
& +\left(m_{L}^{2}\right)_{i}^{j} \tilde{l}^{\dagger i} \tilde{l}_{j}+\left(m_{E}^{2}\right)_{i}^{j} \tilde{e}^{\dagger i} \tilde{e}_{j}+\Delta_{1}^{2} h_{1}^{\dagger} h_{1}+\Delta_{2}^{2} h_{2}^{\dagger} h_{2} \\
& +\left\{A_{D}^{i j} \tilde{q}_{i} \tilde{d}_{j} h_{1}+A_{L}^{i j} \tilde{e}_{i} \tilde{l}_{j} h_{1}+A_{U}^{i j} \tilde{q}_{i} \tilde{u}_{j} h_{2}-B \mu h_{1} h_{2}+\text { h. c. }\right\} \\
& +\frac{1}{2}\left\{M_{1} \tilde{B} \tilde{B}+M_{2} \tilde{W} \tilde{W}+M_{3} \tilde{G} \tilde{G}+\text { h. c. }\right\}
\end{aligned}
$$

where $\tilde{q}_{i}, \tilde{d}_{i}, \tilde{u}_{i}, \tilde{e}_{i}, \tilde{l}_{i}, h_{1}$ and $h_{2}$ are scalar components of $Q_{i}, D_{i}, U_{i}, E_{i}, L_{i}, H_{1}$ and $H_{2}$, respectively, and $\tilde{B}, \tilde{W}$ and $\tilde{G}$ are $\mathrm{U}(1), \mathrm{SU}(2)$ and $\mathrm{SU}(3)$ gauge fermion fields (bino, wino and gluino), respectively. $\mathrm{SU}(2)$ and $\mathrm{SU}(3)$ suffices are omitted in (2.3) 
for simplicity. We assume that the soft SUSY breaking parameters satisfy simple relations at the GUT scale:

$$
\begin{aligned}
\left(m_{Q}^{2}\right)_{i}^{j} & =\left(m_{D}^{2}\right)_{i}^{j}=\left(m_{U}^{2}\right)_{i}^{j}=\left(m_{L}^{2}\right)_{i}^{j}=\left(m_{E}^{2}\right)_{i}^{j} \equiv m_{0}^{2} \delta_{i}^{j} \\
\Delta_{1}^{2} & =\Delta_{2}^{2}=m_{0}^{2}, \\
A_{D}^{i j} & =f_{D X}^{i j} A_{X} m_{0}, A_{L}^{i j}=f_{L X}^{i j} A_{X} m_{0}, A_{U}^{i j}=g_{U X}^{i j} A_{X} m_{0} \\
M_{1} & =M_{2}=M_{3} \equiv M_{g X}
\end{aligned}
$$

where the suffix " $X$ " stands for the value at the GUT scale. The boundary conditions (2.4) are due to the minimal SUGRA model, where local SUSY is spontaneously broken in the hidden sector which couples to the observable sector (SUSY-GUT, in the present case) only gravitationally, and hence universal soft SUSY breaking terms are induced in the observable sector [8].

Below the GUT scale, radiative corrections modify all parameters in the superpotential (2.2) and the soft SUSY breaking terms (2.3), as well as three gauge coupling constants $g_{1}, g_{2}$ and $g_{3}$ for $\mathrm{U}(1), \mathrm{SU}(2)$ and $\mathrm{SU}(3)$, respectively. The evolution of the parameters are described by the RGEs [11]. According to the radiative $\mathrm{SU}(2) \times \mathrm{U}(1)$ breaking scenario [10], we numerically solve the RGEs down to the electroweak scale $m_{Z}$ and evaluate the effective potential for the neutral Higgs fields:

$$
\begin{aligned}
V(\text { Higgs })= & V_{0}+V_{1}, \\
V_{0}= & \left(\mu^{2}+\Delta_{1}^{2}\right)\left|h_{1}\right|^{2}+\left(\mu^{2}+\Delta_{2}^{2}\right)\left|h_{2}\right|^{2}-\left(B \mu h_{1} h_{2}+\text { h. с. }\right) \\
& +\frac{g_{1}^{2}+g_{2}^{2}}{8}\left(\left|h_{1}\right|^{2}-\left|h_{2}\right|^{2}\right)^{2}, \\
V_{1}= & \frac{1}{64 \pi^{2}} \operatorname{Str} \mathcal{M}^{4}\left(\log \frac{\mathcal{M}^{2}}{m_{Z}^{2}}-\frac{3}{2}\right),
\end{aligned}
$$

where Str means the supertrace and $\mathcal{M}$ includes all (s)quark and (s)lepton masses. Then the electroweak symmetry breaking condition

$$
\begin{aligned}
\left\langle h_{1}\right\rangle & =v \cos \beta, \quad\left\langle h_{2}\right\rangle=v \sin \beta \\
m_{Z}^{2} & =\frac{g_{2}^{2}}{2 \cos ^{2} \theta_{W}} v^{2},
\end{aligned}
$$

is imposed. 


\section{Flavor mixing in the gluino coupling}

In order to discuss the flavor mixing in the gluino coupling, we have to diagonalize the mass matrices for quarks and squarks. Throughout the calculation hereafter, we choose the basis in the generation space for the superfields so that the Yukawa coupling constants for up-type quarks and leptons should be diagonalized at the electroweak scale. The Yukawa terms in (2.2) are then written as

$$
W_{\text {Yukawa }}\left(m_{Z}\right)=\hat{f}_{D}^{k j}\left(V_{\mathrm{KM}}^{\dagger}\right)_{k}^{i} Q_{i} D_{j} H_{1}+\hat{f}_{L}^{i j} E_{i} L_{j} H_{1}+\hat{g}_{U}^{i j} Q_{i} U_{j} H_{2},
$$

where the notation "^" stands for a diagonal matrix and $V_{\mathrm{KM}}$ is the KobayashiMaskawa matrix. All eigenvalues of $\hat{f}_{D}, \hat{f}_{L}$ and $\hat{g}_{U}$ are taken to be real positive. Since this choice of the basis is different from that in the GUT superpotential (2.1), a re-diagonalization of the Yukawa couplings at the GUT scale is needed in order to find the unification condition of $f_{D}$ and $f_{L}$ and the relation between the Yukawa coupling constants and the dimension-five coupling constants $C_{L, R}$. $W_{\text {Yukawa }}$ at the GUT scale is diagonalized with appropriate unitary matrices $U_{Q}^{(f)}, U_{D}, U_{E}, U_{L}, U_{Q}^{(g)}$ and $U_{U}$ :

$$
\begin{aligned}
W_{\text {Yukawa }}\left(M_{X}\right)= & f_{D X}^{i j} Q_{i} D_{j} H_{1}+f_{L X}^{i j} E_{i} L_{j} H_{1}+g_{U X}^{i j} Q_{i} U_{j} H_{2} \\
= & \hat{f}_{D X}^{k l}\left(U_{Q}^{(f)}\right)_{k}^{i}\left(U_{D}\right)_{l}^{j} Q_{i} D_{j} H_{1}+\hat{f}_{L X}^{k l}\left(U_{E}\right)_{k}{ }^{i}\left(U_{L}\right)_{l}^{j} E_{i} L_{j} H_{1} \\
& +\hat{g}_{U X}^{k l}\left(U_{Q}^{(g)}\right)_{k}^{i}\left(U_{U}\right)_{l}^{j} Q_{i} U_{j} H_{2} .
\end{aligned}
$$

The unification condition of $f_{D}$ and $f_{L}$ is then written as

$$
\hat{f}_{D X}^{i j}=\hat{f}_{L X}^{i j}
$$

The matter multiplets are accommodated into the $\mathrm{SU}(5)$ multiptets as

$$
\begin{aligned}
\Psi_{i} & \Leftarrow\left\{Q_{i}, \quad\left(U_{Q}^{(g) \dagger} P^{\dagger} U_{U}\right)_{i}^{j} U_{j}, \quad\left(U_{Q}^{(f) \dagger} U_{E}\right)_{i}^{j} E_{j}\right\}, \\
\Phi_{i} & \Leftarrow\left\{D_{i}, \quad\left(U_{D}^{\dagger} U_{L}\right)_{i}^{j} L_{j}\right\},
\end{aligned}
$$

†The "unification" of $\hat{f}_{D X}$ and $\hat{f}_{L X}$, with quark masses given in Ref. [12], however, is not so satisfactory numerically in the first and the second generations as the gauge coupling unification. We ignore the difference in the present calculation, since we may still have ambiguities of the renormalization effect in the very low energy region. 
and the GUT Yukawa coupling constants in Eq. (2.1) are expressed with those in Eq. (3.2a) as

$$
\begin{aligned}
f^{i j} & =f_{D X}^{i j} \\
g^{i j} & =g_{U X}^{i k}\left(U_{U}^{\dagger} P U_{Q}^{(g)}\right)_{k}^{j}
\end{aligned}
$$

where $P$ is a diagonal phase matrix which cannot be absorbed by field redefinitions in the colored Higgs coupling [13].

The origin of the flavor mixing in the gluino coupling lies in the difference between the mass basis for quarks and that for squarks. The mass matrix for uptype squarks is expressed as

$$
\begin{aligned}
-\mathcal{L}(\text { s-up mass }) & =\left(\tilde{q}_{u}, \tilde{u}^{\dagger}\right) \mathcal{M}_{\tilde{u}}^{2}\left(\begin{array}{c}
\tilde{q}_{u}^{\dagger} \\
\tilde{u}
\end{array}\right) \\
& =\left(\tilde{q}_{u i}, \tilde{u}^{\dagger i}\right)\left(\begin{array}{cc}
\left(m_{L L}^{2}\right)_{j}^{i} & \left(m_{L R}^{2}\right)^{i j} \\
\left(m_{R L}^{2}\right)_{i j} & \left(m_{R R}^{2}\right)_{i}^{j}
\end{array}\right)\left(\begin{array}{c}
\tilde{q}_{u}^{\dagger j} \\
\tilde{u}_{j}
\end{array}\right), \\
\left(m_{L L}^{2}\right)_{j}^{i} & =\left(M_{U} M_{U}^{\dagger}\right)_{j}^{i}+\left(m_{Q}^{2}\right)_{j}^{i}+m_{W}^{2} \cos 2 \beta\left(\frac{1}{2}-\frac{1}{6} \tan ^{2} \theta_{W}\right) \delta_{j}^{i} \\
\left(m_{R R}^{2}\right)_{i}^{j} & =\left(M_{U}^{\dagger} M_{U}\right)_{i}^{j}+\left(m_{U}^{2}\right)_{i}^{j}+m_{W}^{2} \cos 2 \beta\left(\frac{2}{3} \tan ^{2} \theta_{W}\right) \delta_{i}^{j} \\
\left(m_{L R}^{2}\right)^{i j} & =\mu M_{U}^{i j} \cot \beta+A_{U}^{i j} v \sin \beta \\
m_{R L} & =m_{L R}^{\dagger},
\end{aligned}
$$

where $M_{U}$ is the up-type quark mass matrix $M_{U}^{i j}=g_{U}^{i j} v \sin \beta$ and $\tilde{q}_{u}$ is the up-type component of the $\mathrm{SU}(2)$ doublet $\tilde{q}$. The squark mass matrix $\mathcal{M}_{\tilde{u}}^{2}$ is not diagonalized with the quark mass basis (3.1) since off-diagonal elements are induced in the soft SUSY breaking parameter matrices due to the renormalization effect. Squark mass basis is obtained by diagonalizing (3.6) with a $6 \times 6$ unitary matrix $\tilde{U}_{U}$ :

$$
\begin{aligned}
\tilde{u}_{I}^{\prime} & =\left(\tilde{U}_{U}\right)_{I}^{J} \tilde{u}_{J}, I=1,2, \cdots, 6, \\
\tilde{u}_{I} & =\left\{\begin{array}{ll}
\tilde{q}_{u I} & \text { for } I=1,2,3 \\
\tilde{u}_{I-3} & \text { for } I=4,5,6
\end{array},\right. \\
\tilde{U}_{U}^{\dagger} \mathcal{M}_{\tilde{u}}^{2} \tilde{U}_{U} & =\text { diagonal },
\end{aligned}
$$

where $\tilde{u}_{I}^{\prime}$ is the mass eigenstate of up-type squark. We define the numbering of $\tilde{u}_{I}^{\prime}$ such that the mixing of $\tilde{u}_{I}$ is the largest in $\tilde{u}_{I}^{\prime}$. Accordingly we call $\tilde{u}_{1}^{\prime}, \tilde{u}_{2}^{\prime}, \cdots, \tilde{u}_{6}^{\prime}$ 
as $\tilde{u}_{L}, \tilde{c}_{L}, \tilde{t}_{L}, \tilde{u}_{R}, \tilde{c}_{R}$ and $\tilde{t}_{R}$ respectively in the later discussions. The mass bases of down-type squarks and charged sleptons are obtained in the same way with $6 \times 6$ unitary matrices $\tilde{U}_{D}$ and $\tilde{U}_{E}$, respectively. Notice that no generation mixing occurs in the lepton/slepton sector since the right-handed (s)neutrino does not exist in the minimal model; the nonvanishing off-diagonal elements of the slepton mass matrix are left-right mixing components only. Consequently, quark-squark-gluino coupling is written as

$$
\begin{aligned}
\left.\mathcal{L}_{\text {int }} \text { (gluino }\right)=-i \sqrt{2} g_{3}\left\{\tilde{d}^{\prime \dagger I}\left(\left(\tilde{U}_{D}\right)_{I}^{k}\left(V_{\mathrm{KM}}\right)_{k}^{j} \tilde{G} d_{L j}+\left(\tilde{U}_{D}\right)_{I}^{j+3} \overline{\tilde{G}} \bar{d}_{R j}\right)\right. \\
\left.+\tilde{u}^{\prime \dagger I}\left(\left(\tilde{U}_{U}\right)_{I}^{j} \tilde{G} u_{L j}+\left(\tilde{U}_{U}\right)_{I}^{j+3} \overline{\tilde{G}} \bar{u}_{R j}\right)\right\}+ \text { h. c. } \\
=-i \sqrt{2} g_{3}\left\{\tilde{d}^{\prime \dagger I}\left(\left(\tilde{U}_{D}^{\prime}\right)_{I}^{j} \tilde{G} d_{L j}+\left(\tilde{U}_{D}\right)_{I}^{j+3} \overline{\tilde{G}} \bar{d}_{R j}\right)\right. \\
\left.+\tilde{u}^{\prime \dagger I}\left(\left(\tilde{U}_{U}\right)_{I}^{j} \tilde{G} u_{L j}+\left(\tilde{U}_{U}\right)_{I}^{j+3} \overline{\tilde{G}} \bar{u}_{R j}\right)\right\}+ \text { h. c. }
\end{aligned}
$$

with the definition of $\tilde{U}_{D}^{\prime}$ as

$$
\left(\tilde{U}_{D}^{\prime}\right)_{I}^{j} \equiv\left(\tilde{U}_{D}\right)_{I}^{k}\left(V_{\mathrm{KM}}\right)_{k}^{j}
$$

$u_{L i}$ and $d_{L i}$ in (3.8) are left-handed quarks of mass eigenstates which compose the $\mathrm{SU}(2)$ doublet as

$$
Q_{i} \ni\left(\begin{array}{c}
u_{L i} \\
\left(V_{\mathrm{KM}}\right)_{i}^{j} d_{L j}
\end{array}\right),
$$

and $u_{R i}$ and $d_{R i}$ are the fermion components of $U_{i}$ and $D_{i}$, respectively. Similar flavor mixing formulae are obtained for other gaugino (wino and bino) coupling terms.

\section{Nucleon decay with dimension five operators}

As mentioned in Sec. [1], the nucleon decay amplitude in the minimal SU(5) SUSYGUT model is dominated by the dimension five operators [2] induced by colored higgsino/Higgs exchanges. Since the dimension five operators are made from two fermion (quark/lepton) and two boson (squark/slepton) component fields, effective baryon number violating four-fermion operators are generated by one loop "dressing" diagrams which involve gauginos or higgsinos (see Fig. 1). In the present 
calculation, only the gluino dressing and the charged wino dressing diagrams are included; contributions from higgsino dressing diagrams are negligibly small due to the small Yukawa couplings of light quarks $(u, d, s)$, compared to the $\mathrm{SU}(2)$ gauge coupling $g_{2}$; neutral wino and bino coupling have the same flavor mixing structure as that in the gluino coupling, hence their contributions are smaller than that from the gluino dressing.

The dimension five coupling constants $C_{L}$ and $C_{R}$ of (2.2) at the GUT scale are written in terms of the Yukawa coupling constants (see (3.4) and (3.5)):

$$
\begin{aligned}
& C_{L X}^{i j k l}=f_{D X}^{i m}\left(U_{D}^{\dagger} U_{L}\right)_{m}^{j} g_{U X}^{k n}\left(U_{U}^{\dagger} P U_{Q}^{(g)}\right)_{n}^{l}, \\
& C_{R X}^{i j k l}=f_{D X}^{m j}\left(U_{Q}^{(g) \dagger} P^{\dagger} U_{U}\right)_{m}^{i} g_{U X}^{n l}\left(U_{Q}^{(f) \dagger} U_{E}\right)_{n}^{k} .
\end{aligned}
$$

Note that this relation with the index " $X$ " removed does not hold true at the electroweak scale. The effective baryon number violating four-fermion operators at the electroweak scale are written as

$$
\begin{aligned}
\mathcal{L}_{\text {eff }}(\Delta B= \pm 1)= & \left(\tilde{C}_{\nu}^{i j k l}(\tilde{G})+\tilde{C}_{\nu}^{i j k l}(\tilde{W})\right) \epsilon_{a b c}\left(u_{L k}^{a} d_{L l}^{b}\right)\left(d_{L i}^{c} \nu_{L j}\right) \\
& +\left(\tilde{C}_{e}^{i j k l}(\tilde{G})+\tilde{C}_{e}^{i j k l}(\tilde{W})\right) \epsilon_{a b c}\left(u_{L k}^{a} d_{L l}^{b}\right)\left(u_{L i}^{c} e_{L j}\right) \\
& +(\text { right-handed quark/lepton })+\text { h. c. }
\end{aligned}
$$

where $\tilde{C}_{\nu, e}$ are calculated as follows with use of the numerical values of $C_{L}$ and $C_{R}$ at the electroweak scale to be obtained through their RGEs, squark/slepton mass eigenvalues and the mixing matrices in the gaugino couplings ${ }^{\ddagger}$ :

$$
\begin{aligned}
& \tilde{C}_{\nu}^{i j k l}(\tilde{G})=-\frac{4 g_{3}^{2}}{3 M_{C}}\left\{\left(C_{L}^{i j m n}-C_{L}^{n j m i}\right)\left(\tilde{U}_{U}^{\dagger}\right)_{m}^{I}\left(\tilde{U}_{D}^{\dagger}\right)_{n}^{J} F_{\tilde{G}}\left(\tilde{u}_{I}^{\prime}, \tilde{d}_{J}^{\prime}\right)\left(\tilde{U}_{U}\right)_{I}^{k}\left(\tilde{U}_{D}^{\prime}\right)_{J}^{l}\right. \\
& \left.-\left(C_{L}^{m j k n}-C_{L}^{n j k m}\right)\left(\tilde{U}_{D}^{\dagger}\right)_{m}^{I}\left(\tilde{U}_{D}^{\dagger}\right)_{n}^{J} F_{\tilde{G}}\left(\tilde{d}_{I}^{\prime}, \tilde{d}_{J}^{\prime}\right)\left(\tilde{U}_{D}^{\prime}\right)_{I}^{i}\left(\tilde{U}_{D}^{\prime}\right)_{J}^{l}\right\}, \\
& \tilde{C}_{\nu}^{i j k l}(\tilde{W})=-\frac{g_{2}^{2}}{M_{C}}\left\{\left(C_{L}^{i j m n}-C_{L}^{n j m i}\right)\left(\tilde{U}_{U}^{\dagger}\right)_{m}^{I}\left(\tilde{U}_{D}^{\dagger}\right)_{n}^{J} F_{\tilde{W}}\left(\tilde{u}_{I}^{\prime}, \tilde{d}_{J}^{\prime}\right)\left(\tilde{U}_{U}^{\prime}\right)_{I}^{k}\left(\tilde{U}_{D}\right)_{J}^{l}\right. \\
& \left.+\left(C_{L}^{m n k l}-C_{L}^{k n m l}\right)\left(\tilde{U}_{U}^{\dagger}\right)_{m}^{I}\left(\tilde{U}_{E}^{\dagger}\right)_{n}^{J} F_{\tilde{W}}\left(\tilde{u}_{I}^{\prime}, \tilde{e}_{J}^{\prime}\right)\left(\tilde{U}_{U}^{\prime}\right)_{I}^{i}\left(\tilde{U}_{E}\right)_{J}^{j}\right\}, \\
& \tilde{C}_{e}^{i j k l}(\tilde{G})=-\frac{4 g_{3}^{2}}{3 M_{C}}\left\{\left(C_{L}^{i j m n}-C_{L}^{m j i n}\right)\left(\tilde{U}_{U}^{\dagger}\right)_{m}^{I}\left(\tilde{U}_{D}^{\dagger}\right)_{n}^{J} F_{\tilde{G}}\left(\tilde{u}_{I}^{\prime}, \tilde{d}_{J}^{\prime}\right)\left(\tilde{U}_{U}\right)_{I}^{k}\left(\tilde{U}_{D}^{\prime}\right)_{J}^{l}\right.
\end{aligned}
$$

${ }^{\ddagger}$ Contributions from $C_{R}$ ’s and higgsino/neutralino dressings are estimated to be small and neglected in the present calculations. 


$$
\begin{aligned}
&\left.-\left(C_{L}^{m j n l}-C_{L}^{n j m l}\right)\left(\tilde{U}_{U}^{\dagger}\right)_{m}^{I}\left(\tilde{U}_{U}^{\dagger}\right)_{n}^{J} F_{\tilde{G}}\left(\tilde{u}_{I}^{\prime}, \tilde{u}_{J}^{\prime}\right)\left(\tilde{U}_{U}\right)_{I}^{i}\left(\tilde{U}_{U}\right)_{J}^{k}\right\}, \\
& \tilde{C}_{e}^{i j k l}(\tilde{W})=-\frac{g_{2}^{2}}{M_{C}}\left\{\left(C_{L}^{i j m n}-C_{L}^{m j i n}\right)\left(\tilde{U}_{U}^{\dagger}\right)_{m}^{I}\left(\tilde{U}_{D}^{\dagger}\right)_{n}^{J} F_{\tilde{W}}\left(\tilde{u}_{I}^{\prime}, \tilde{d}_{J}^{\prime}\right)\left(\tilde{U}_{U}^{\prime}\right)_{I}^{l}\left(\tilde{U}_{D}\right)_{J}^{k}\right. \\
&\left.+\left(C_{L}^{m j k l}-C_{L}^{l j k m}\right)\left(\tilde{U}_{D}^{\dagger}\right)_{m}^{I} F_{\tilde{W}}\left(\tilde{d}_{I}^{\prime}, \tilde{\nu}_{j}^{\prime}\right)\left(\tilde{U}_{D}\right)_{I}^{i}\right\} .
\end{aligned}
$$

Here, $\left(\tilde{U}_{D}^{\prime}\right)_{I}^{i}$ is defined in $(3.9)$ and $\left(\tilde{U}_{U}^{\prime}\right)_{I}^{i}=\left(\tilde{U}_{U}\right)_{I}^{j}\left(V_{\mathrm{KM}}\right)_{j}^{i} . F_{\tilde{G}}$ and $F_{\tilde{W}}$ are obtained by the loop integral [3, 4, 5]:

$$
\begin{aligned}
F_{\tilde{G}}\left(\tilde{f}_{1}, \tilde{f}_{2}\right) & =\tilde{F}\left(m_{\tilde{f}_{1}}, m_{\tilde{f}_{2}} ; M_{3}\right), \\
F_{\tilde{W}}\left(\tilde{f}_{1}, \tilde{f}_{2}\right) & =\left(U_{-}\right)_{1}^{\alpha} \tilde{F}\left(m_{\tilde{f}_{1}}, m_{\tilde{f}_{2}} ; M_{ \pm}^{\alpha}\right)\left(U_{+}^{\dagger}\right)_{\alpha}^{1}, \\
\tilde{F}\left(m_{1}, m_{2} ; M\right) & =\frac{1}{16 \pi^{2}} \frac{M}{m_{1}^{2}-m_{2}^{2}}\left(\frac{m_{1}^{2}}{m_{1}^{2}-M^{2}} \log \frac{m_{1}^{2}}{M^{2}}-\frac{m_{2}^{2}}{m_{2}^{2}-M^{2}} \log \frac{m_{2}^{2}}{M^{2}}\right),
\end{aligned}
$$

where $U_{-}, U_{+}$are $2 \times 2$ unitary matrices which diagonalize the chargino mass matrix and $M_{ \pm}^{\alpha}(\alpha=1,2)$ are its eigenvalues:

$$
\begin{aligned}
M(\text { chargino }) & =\left(\begin{array}{cc}
M_{2} & \sqrt{2} m_{W} \sin \beta \\
-\sqrt{2} m_{W} \cos \beta & -\mu
\end{array}\right) \\
& =U_{-}\left(\begin{array}{cc}
M_{ \pm}^{1} & 0 \\
0 & M_{ \pm}^{2}
\end{array}\right) U_{+}^{\dagger} .
\end{aligned}
$$

The low energy QCD correction between $m_{Z}$ and $1 \mathrm{GeV}$ is taken into account in order to evaluate the four fermion operators in the next section. The quark Lagrangian at $\sim 1 \mathrm{GeV}$ is then converted to the hadron chiral Lagrangian with $\Delta B= \pm 1$ terms [3, 14] with use of the matrix element

$$
\left\langle 0\left|\epsilon_{a b c}\left(d_{L}^{a} u_{L}^{b}\right) u_{L}^{c}\right| p\right\rangle=\beta_{p} N_{L}
$$

where $N_{L}$ is a left-handed proton wave function; it enables us to evaluate the partial lifetimes of the nucleon decay.

\section{Numerical calculations}

According to the framework described in the previous sections, we calculate the flavor (and left-right) mixing in gaugino couplings and the nucleon partial lifetimes in a five-dimensional parameter space $\left\{m_{\mathrm{top}}, \tan \beta, m_{0}, M_{g X}, \tilde{A}_{X}\right\}$, where a dimensionful $A$ parameter is defined as $\tilde{A}_{X} \equiv A_{X} m_{0}$. Actual calculations are made 
in the following procedure. At first, $m_{\text {top }}$ and $\tan \beta$ (at the electroweak scale) are fixed. Using the numerical values of light quark masses and the Kobayashi-Maskawa mixing angles given in literatures [12, 15] with the above fixed $m_{\text {top }}$ and $\tan \beta$, we evaluate the Yukawa coupling constants at the electroweak scale (3.1). QCD corrections below the electroweak scale for quark masses other than $m_{\text {top }}$ are included at the one-loop level. Next, the RGEs for the dimensionless parameters i.e., the gauge coupling constants and the Yukawa coupling constants are solved upward to the GUT scale with the boundary conditions at the electroweak scale. At the GUT scale, the Yukawa coupling constants are re-diagonalized to obtain the boundary conditions for the dimension-five coupling constants (4.1). Then the RGEs for the soft SUSY breaking parameters and dimension-five coupling constants are solved downward with the boundary conditions (2.4) and (4.1). Since the RGEs are linear for the dimensionful parameters, all soft SUSY breaking parameters at the electroweak scale are written as linear combinations of the initial parameters $\left(m_{0}, M_{g X}, \tilde{A}_{X}\right)$ 41]:

$$
\begin{aligned}
& \tilde{m}_{I}^{2}\left(m_{Z}\right)=c_{1 I} m_{0}^{2}+c_{2 I} M_{g X}^{2}+c_{3 I} \tilde{A}_{X}^{2}+c_{4 I} M_{g X} \tilde{A}_{X} \\
& \tilde{M}_{J}\left(m_{Z}\right)=d_{1 J} M_{g X}+d_{2 J} \tilde{A}_{X}
\end{aligned}
$$

where $\tilde{m}_{I}^{2}$ and $\tilde{M}_{J}$ are collective notations for the soft SUSY breaking parameters of mass dimension two $\left(m_{Q, D, U, L, E}^{2}, \Delta_{1,2}^{2}\right)$ and one $\left(M_{1,2,3}, A_{D, U, L}\right)$, respectively. The coefficients $c$ 's and $d$ 's are implicit functions of the gauge couplings and the Yukawa couplings and are determined numerically by solving the RGEs with four cases of boundary conditions $\left(m_{0}, M_{g X}, \tilde{A}_{X}\right)=(1,0,0),(0,1,0),(0,0,1)$ and $(0,1,1)$. Once the coefficients are obtained, the values of soft SUSY breaking parameters at the electroweak scale for given $\left(m_{0}, M_{g X}, \tilde{A}_{X}\right)$ are evaluated with the formulae (5.1), and it is easy to scan the three-dimensional parameter space $\left\{m_{0}, M_{g X}, \tilde{A}_{X}\right\}$ for fixed $m_{\text {top }}$ and $\tan \beta$ with this method. The next step is to evaluate the remaining two parameters $\mu$ and $B$ with the electroelectroweak $\mathrm{SU}(2)$ $\times \mathrm{U}(1)$ symmetry breaking condition. The requirement that the minimum of the Higgs potential (2.5) gives the vacuum expectation values (2.6) leads to

$$
\mu^{2}=\frac{\Delta_{2}^{2}-\Delta_{1}^{2}}{2 \cos 2 \beta}-\frac{\Delta_{1}^{2}+\Delta_{2}^{2}}{2}-\frac{1}{2} m_{Z}^{2}
$$




$$
\begin{aligned}
& -\left.\frac{1}{v \cos 2 \beta}\left(\frac{\partial V_{1}}{\partial h_{1}} \cos \beta-\frac{\partial V_{1}}{\partial h_{2}} \sin \beta\right)\right|_{\substack{h_{1}=v \cos \beta \\
h_{2}=v \sin \beta}}, \\
B \mu= & \left(\mu^{2}+\frac{\Delta_{1}^{2}+\Delta_{2}^{2}}{2}\right) \sin 2 \beta \\
& +\left.\frac{1}{v}\left(\frac{\partial V_{1}}{\partial h_{1}} \sin \beta+\frac{\partial V_{1}}{\partial h_{2}} \cos \beta\right)\right|_{\substack{h_{1}=v \cos \beta \\
h_{2}=v \sin \beta}} .
\end{aligned}
$$

Notice that the solution of the equation (5.2) for $\mu$ cannot be written in a simple formula since the one-loop part of the Higgs potential $V_{1}$ depends on $\mu$. We solve (5.2) numerically for both signs of $\mu$ and then calculate $B$ with (5.3). Since all the MSSM parameters and the dimension-five coupling constants at the electroweak scale for a given parameter set $\left(m_{\mathrm{top}}, \tan \beta, m_{0}, M_{g X}, \tilde{A}_{X}\right)$ are thus determined, the mass spectrum of all superparticles and the mixing matrices in the gaugino couplings are obtained by diagonalizing their mass matrices.

We investigate the parameter space $\left\{m_{0}, M_{g X}, A_{X}\right\}$ within the range $10 \mathrm{GeV} \leq$ $m_{0} \leq 10 \mathrm{TeV}, 10 \mathrm{GeV} \leq M_{g X} \leq 10 \mathrm{TeV}$ and $-5 \leq A_{X} \leq+5$ for each combination of $m_{\mathrm{top}}=120,150$ or $180 \mathrm{GeV}$ and $\tan \beta=2,10,30$ or 50 . For $m_{\text {top }}=180$ $\mathrm{GeV}$ and $\tan \beta=2$, the top Yukawa coupling diverges below the GUT scale when solving the RGEs. For $\tan \beta=50$, no consistent radiative breaking solution is found for any $m_{\text {top }}$. Figs. 2a - 2t are histograms for the specified off-diagonal elements of the gluino coupling matrices $\tilde{U}_{U}$ and $\tilde{U}_{D}^{\prime}$ for $m_{\text {top }}=150 \mathrm{GeV}$. The magnitudes of the generation mixing in the right-right and left-right sectors are small compared to the corresponding left-left elements. For a small $\tan \beta$, the left-left elements of $\tilde{U}_{D}^{\prime}$ are approximately equal to the Kobayashi-Maskawa matrix elements: $\left(\tilde{U}_{D}^{\prime}\right)_{2}^{1} \approx V_{c d}$, $\left(\tilde{U}_{D}^{\prime}\right)_{3}^{1} \approx V_{t d}$, etc. in most of the parameter space, while the corresponding offdiagonal elements of $\tilde{U}_{U}$ are small: the mass matrices of up-type quarks, up-type squarks and down-type squarks are diagonalized in the same basis. This agrees with the conclusion of Ref. [11] where those mixing matrices are semi-analytically obtained with an assumption that the bottom Yukawa coupling is much smaller than the top Yukawa coupling, which is applicable for small tan $\beta$. On the other hand, for a large $\tan \beta$, we find that nonvanishing off-diagonal elements in the left-left sector of $\tilde{U}_{U}$ arises with the same order of magnitudes as $V_{\mathrm{KM}}$, which contributes significantly to the charged lepton decay modes (see below). Off-diagonal elements of $\tilde{U}_{D}^{\prime}$ are smaller than those for small $\tan \beta$. The qualitative behavior of the mixing 
matrices is rather independent of the different values of $m_{\mathrm{top}}$.

Using the obtained values of superpartner masses and mixing matrices, we evaluate $\tilde{C}^{\prime}$ 's in (4.2) with the formula (4.3). We then take into account of the low energy QCD correction to $\tilde{C}$ 's at the one-loop level]. We take the chiral Lagrangian factors given in Ref. [3] to derive amplitudes of various decay modes from (4.2). A large uncertainty of the nucleon lifetime comes from the numerical values of $\beta_{p}$ and $M_{C} \cdot \beta_{p}$ is calculated with various methods [17], which give

$$
0.003 \mathrm{GeV}^{3} \leq \beta_{p} \leq 0.03 \mathrm{GeV}^{3}
$$

and Ref. [3, 18] shows

$$
2.2 \times 10^{13} \mathrm{GeV} \leq M_{C} \leq 2.3 \times 10^{17} \mathrm{GeV}
$$

Here, we take a small value of $\beta_{p}=0.003 \mathrm{GeV}^{3}$ and a large value of $M_{C}=10^{17}$ $\mathrm{GeV}$ so that we have a longer nucleon lifetime for the safety of the later arguments. Fig. 3 shows the partial lifetime for each nucleon decay mode with fixed $m_{\text {top }}=150$ $\mathrm{GeV}$ and $\tan \beta=2$. The range of each lifetime comes mainly from the ranges of the soft SUSY breaking parameters. As can be seen in the figure, $K \bar{\nu}$ decay modes are dominant and most severely constrained by the experiments [19, 20] as

$$
\begin{array}{llr}
\tau\left(p \rightarrow K^{+} \bar{\nu}\right) \geq 1.0 \times 10^{32} \mathrm{yrs} & (\mathrm{KAMIOKANDE}) \\
\tau\left(p \rightarrow K^{+} \bar{\nu}\right) \geq 6.2 \times 10^{31} \mathrm{yrs} & (\mathrm{IMB}) \\
\tau\left(n \rightarrow K^{0} \bar{\nu}\right) \geq 8.6 \times 10^{31} \mathrm{yrs} & (\mathrm{KAMIOKANDE)}, \\
\tau\left(n \rightarrow K^{0} \bar{\nu}\right) \geq 1.5 \times 10^{31} \mathrm{yrs} & (\mathrm{IMB})
\end{array}
$$

Our main concern is to study the contribution of the gluino dressing diagrams. To do that, we compare $\tau$ (wino) with $\tau$ (total), where $\tau$ (wino) is a partial lifetime calculated with only the wino dressing diagrams taken into account and $\tau$ (total) is that calculated with both wino and gluino dressing diagrams combined. The results for $m_{\text {top }}=150 \mathrm{GeV}$ are presented in Figs. 4a -40 . The nucleon decay amplitude is dominated by the wino dressing diagrams if $\tau$ (wino) $/ \tau$ (total) $\approx 1$, while it is dominated by the gluino dressing diagrams if $\tau$ (wino) $/ \tau($ total $) \gg 1$. There occur cancellations between the wino dressing contributions and gluino dressing contributions in $\tau($ wino $) / \tau($ total $) \ll 1$ region. The gluino contributions are small

\footnotetext{
$\S$ This corresponds to taking $A_{\mathrm{L}} \approx 0.46$, where $A_{\mathrm{L}}$ is the low energy QCD factor used in literatures [3, 4, 5, 6, 16]. It is argued the two-loop analysis gives $A_{\mathrm{L}} \approx 0.28$ in Ref. [16]
} 
for any modes in the small $\tan \beta$ case. For large $\tan \beta$, however, gluino dominant region is realized in the charged lepton modes. This is brought about by the following two reasons: (1) in the simplified analyses based on an assumption of the diagonal gluino coupling, the gluino dressing diagrams contribute only to the $K \bar{\nu}$ modes due to the color antisymmetry in the dimension-five coupling [5, 6]. In the present case, the gluino coupling is not diagonal any more and hence the gluino dressing processes contribute to the decay modes other than $K \bar{\nu}$ modes. The nonvanishing off-diagonal gluino coupling in the up sector for large $\tan \beta$ significantly contributes to the charged lepton modes. (2) furthermore, if the squark masses are much larger than the gaugino masses, the amplitude from the gluino dressing diagrams is enhanced by a factor of $\left(\alpha_{3}\left(m_{Z}\right) / \alpha_{2}\left(m_{Z}\right)\right)^{2}$ compared to that from the wino dressing diagrams, since $\tilde{F}$ in Eq. (4.4) is asymptotically

$$
\tilde{F}(m, m ; M) \sim \frac{1}{16 \pi^{2}} \frac{M}{m^{2}} \quad \text { for } m \gg M,
$$

and $M_{3}\left(m_{Z}\right) / M_{2}\left(m_{Z}\right)=\alpha_{3}\left(m_{Z}\right) / \alpha_{2}\left(m_{Z}\right)$ because of the GUT relation of gaugino masses. Since (5.4) gives an overall suppression of the nucleon decay amplitude for $m \gg M$ (see Fig. 6b), the dominant gluino contribution in Figs. $4 \mathrm{~b}$ and $4 \mathrm{c}$ is realized in the long lifetime region (see Fig. 5).

Translating the scanned parameters $\left(m_{0}, M_{g X}, A_{X}\right)$ into the MSSM parameters $\left(m_{\tilde{d}_{L}}, M_{2}, \mu\right)$ where we take $m_{\tilde{d}_{L}}$, the down-type squark mass of the first generation as the typical squark mass, we plot the calculated points in the MSSM parameter space for $m_{\mathrm{top}}=150 \mathrm{GeV}$ and $\tan \beta=2$ in Figs. 6a and 6b. The region A in Fig. 6a is excluded by LEP constraints on charginos and neutralinos [21]:

$$
\begin{aligned}
m_{\chi^{ \pm}} & >45 \mathrm{GeV} \\
\Gamma(Z \rightarrow \chi \chi) & <22 \mathrm{MeV} \\
B\left(Z \rightarrow \chi \chi^{\prime}\right) & <5 \times 10^{-5} \\
B\left(Z \rightarrow \chi^{\prime} \chi^{\prime}\right) & <5 \times 10^{-5}
\end{aligned}
$$

where $\chi^{ \pm}$is a chargino, $\chi$ is the lightest neutralino and $\chi^{\prime}$ is a heavier neutralino. No solution with radiative $\mathrm{SU}(2) \times \mathrm{U}(1)$ breaking is found in region $\mathrm{B}$, which is a forbidden region. Points plotted with small dots are excluded due to the present lower bound for the proton lifetime $\tau\left(p \rightarrow K^{+} \bar{\nu}\right)>10^{32}$ yrs, giving the constraint of 
$|\mu| \gtrsim 300 \mathrm{GeV}$. This constraint for $\mu$ is roughly unchanged for different $m_{\mathrm{top}}$ and/or

$\tan \beta$. Fig. 6 b shows the squark mass bound $m_{\tilde{d}_{L}} \gtrsim 400 \mathrm{GeV}$. If the lower bound for the proton lifetime is raised to $\tau\left(p \rightarrow K^{+} \bar{\nu}\right)>10^{33}$ yrs with the near future experiment at Super-KAMIOKANDE, most of the parameter region with $m_{\tilde{d}_{L}} \lesssim$ $1 \mathrm{TeV}$ will be excluded. Lower bounds for other first and second generation squark masses are found similar to that for $m_{\tilde{d}_{L}}$, while the bound for the third generation squarks is lower in general due to the renormalization effect and the left-right mixing. Since the nucleon lifetime is approximately proportional to $(\tan \beta)^{-2}$ [3], the lower bound for the squark mass is raised for larger $\tan \beta$. In fact, $\tau\left(p \rightarrow K^{+} \bar{\nu}\right)>10^{32}$ yrs implies $m_{\tilde{d}_{L}} \gtrsim 1 \mathrm{TeV}$ for $\tan \beta=30$.

\section{Conclusion}

In this paper we have made a systematic analysis of the flavor mixing in the gaugino couplings within the framework of the minimal SUGRA-GUT. We have solved the one-loop RGEs for all MSSM parameters including off-diagonal Higgs coupling matrices with five input parameters, namely $\left(m_{\mathrm{top}}, \tan \beta\right)$ at the electroweak scale $m_{Z}$ and $\left(m_{0}, M_{g X}, A_{X}\right)$ at the GUT scale $M_{X}$, and we have numerically obtained full mass spectra and mixing matrices, which satisfy the radiative electroweak symmetry breaking condition. For a small $\tan \beta(\tan \beta=2)$, we have obtained a result consistent with the semi-analytic study [1], in which the top Yukawa coupling is assumed to be much larger than other Yukawa couplings: the left-left sector of the generation mixing matrix in the down-type quark-squark-gluino coupling is approximately equal to the Kobayashi-Maskawa matrix, while the off-diagonal mixing matrix elements in the up-type gluino coupling are small. On the other hand, for large $\tan \beta=10$ and 30 where the bottom (and tau, for extremely large $\tan \beta$ ) Yukawa coupling is not negligibly small compared with the top Yukawa coupling, we have found that nonvanishing generation mixing in the up-type gluino coupling occurs with the magnitudes comparable to the corresponding Kobayashi-Maskawa matrix elements. The generation mixing in the down-type gluino coupling is also changed considerably.

We have applied the generation mixing to the calculation of nucleon decay widths to study the contributions from the gluino dressing diagrams compared with 
the wino dressing diagrams. In result, it is found that the gluino dressing diagrams give the dominant contribution to the decay mode containing a charged lepton if $\tan \beta \gg 1$ and $M_{3} \ll m_{\tilde{q}}$ (typical squark mass). For the charged lepton modes with small $\tan \beta$, or the (anti-) neutrino emission modes with any $\tan \beta$, the gluino contributions are relatively small. In those cases, the contributions from the gluino dressing are at most of the same order of magnitude as the wino dressing contributions. We have scanned the MSSM parameter space to find allowed regions with the present constraints given by the nucleon decay experiments and the accelerator experiments in. The latter excludes the parameter region of small superpartner masses, and the former gives a strict bound to the masses of first and second generation squarks. We argue that the whole parameter region with $m_{\tilde{d}_{L}} \lesssim 1 \mathrm{TeV}$ in the minimal SU(5) SUGRA-GUT model can be tested by Super-KAMIOKANDE.

Our method of calculations and the numerical result itself are adaptable to the analyses of FCNC in the minimal SUGRA model, which will be discussed elsewhere.

\section{Acknowledgment}

One of the authors (T. G.) would like to thank J. Hisano for helpful discussions.

IIn addition, cosmological constraint will be given by the analyses of the relic abundance of the lightest superprticle [22]. 


\section{References}

[1] P. Langacker and M.-X. Luo, Phys. Rev. D 44, 897 (1991);

U. Amaldi, W. de Boer and H. Fürstenau, Phys. Lett. B 260, 447 (1991);

W.J. Marciano, Annu. Rev. Nucl. Part. 41, 469 (1991).

[2] N. Sakai and T. Yanagida, Nucl. Phys. B197, 533 (1982);

S. Weinberg, Phys. Rev. D 26, 287 (1982).

[3] J. Hisano, H. Murayama and T. Yanagida, Nucl. Phys. B402, 46 (1993).

[4] M. Matsumoto, J. Arafune, H. Tanaka and K. Shiraishi, Phys. Rev. D 46, 3966 (1992).

[5] P. Nath, A.H. Chamseddine and R. Arnowitt, Phys. Rev. D 32, 2348 (1985).

[6] J. Ellis, D.V. Nanopoulos and S. Rudaz, Nucl. Phys. B202, 43 (1982).

[7] For reviews, see H.P. Nilles, Phys. Rep. 110, 1 (1984); B. Lahanas and D.V. Nanopoulos, ibid. 145, 1 (1987), and references therein.

[8] R. Barbieri, S. Ferrara and C.A. Savoy, Phys. Lett. 119B, 343 (1982);

A.H. Chamseddine, R. Arnowitt and P. Nath, Phys. Rev. Lett. 49, 970 (1982);

L. Hall, J. Lykken and S. Weinberg, Phys. Rev. D 27, 2359 (1983).

[9] Y. Yamada, KEK preprint KEK-TH-383 (1994);

S.P. Martin and M.T. Vaughn, Northeastern University preprint NUB-308193TH (1993).

[10] K. Inoue, A. Kakuto, H. Komatsu and S. Takeshita, Prog. Theor. Phys. 71, 413 (1984); ibid. 68, 927 (1982);

L. Alvarez-Gaumé, J. Polchinski and M.B. Wise, Nucl. Phys. B221, 495 (1983).

[11] A. Bouquet, J. Kaplan and C.A. Savoy, Phys. Lett. 148B, 69 (1984); Nucl. Phys. B262, 299 (1985).

[12] J. Gasser and H. Leutwyler, Phys. Rep. 87, 77 (1982).

[13] J. Ellis, M.K. Gaillard and D.V. Nanopoulos, Phys. Lett. B88, 320 (1979).

[14] S. Chadha and M. Daniel, Nucl. Phys. B229, 105 (1983);

M. Claudson, M.B. Wise and L. Hall, Nucl. Phys. B195, 297 (1982).

[15] K. Hikasa et al. , Phys. Rev. D 45, S1 (1992). 
[16] R. Arnowitt and P. Nath, Phys. Rev. Lett. 69, 725 (1992).

[17] M.B. Gavela, S.F. King, C.T. Sachrajda, G. Martinelli, M.L. Paciello and B. Taglienti, Nucl. Phys. B312, 269 (1989);

Y. Hara, S. Itoh, Y. Iwasaki and T. Yoshie, Phys. Rev. D 34, 3399 (1986);

S.J. Brodsky, J. Ellis, J.S. Hagelin and C.T. Sachrajda, Nucl. Phys. B238, 561 (1984).

[18] K. Hagiwara and Y. Yamada, Phys. Rev. Lett. 70, 709 (1993).

[19] K.S. Hirata et al. (KAMIOKANDE Collabolation), Phys. Lett. B 220, 308 (1989).

[20] R. Becker-Szendy et al. (IMB Collabolation), Proceedings of 23rd ICRC, Calgary, 1993, edited by D.A. Leahy, Vol. 4, p. 589 (1993);

T.J. Haines et al. (IMB Collabolation), Phys. Rev. Lett. 57, 1986 (1986).

[21] D. Decamp et al. (ALEPH Collabolation), Phys. Rep. 216, 253 (1992);

O. Adriani et al. (L3 Collabolation), Phys. Rep. 236, 1 (1993);

P. Abreu et al. (DELPHI Collabolation), Nucl. Phys. B367, 511 (1991);

M.Z. Akrawy et al. (OPAL Collabolation), Phys. Lett. B248, 211 (1990); ibid. B240, 261 (1990).

[22] S. Mizuta and M. Yamaguchi, Phys. Lett. B298, 120 (1993);

M. Drees and M.M. Nojiri, Phys. Rev. D 47, 376 (1993);

M. Kawasaki and S. Mizuta, Phys. Rev. D 46, 1634 (1993). 


\section{Figure Captions}

FIG. 1 Examples of dressing diagrams which contribute to the proton decay process $p \rightarrow K^{+} \bar{\nu}$.

FIG. 2a Histograms of the mixing matrix element $\left(\tilde{U}_{U}\right)_{2}^{1}\left(u_{L}-\tilde{c}_{L}\right.$ mixing) for $\tan \beta=2,10$ and 30 with $m_{\text {top }}=150 \mathrm{GeV}, 10 \mathrm{GeV} \leq m_{0} \leq 10 \mathrm{TeV}$, $10 \mathrm{GeV} \leq M_{g X} \leq 10 \mathrm{TeV}$ and $-5 \leq A_{X} \leq+5$.

FIG. 20 Histograms of the mixing matrix element $\left(\tilde{U}_{U}\right)_{3}^{1}\left(u_{L}-\tilde{t}_{L}\right.$ mixing) for $\tan \beta=2,10$ and 30 with $m_{\text {top }}=150 \mathrm{GeV}$. The parameters are same as those in Fig. 2a.

FIG. 20 Histograms of the mixing matrix element $\left(\tilde{U}_{D}^{\prime}\right)_{2}^{1}\left(d_{L}-\tilde{s}_{L}\right.$ mixing) for $\tan \beta=2,10$ and 30 with $m_{\text {top }}=150 \mathrm{GeV}$. The parameters are same as those in Fig. 2a.

FIG. 2d Histograms of the mixing matrix element $\left(\tilde{U}_{D}^{\prime}\right)_{3}^{1}\left(d_{L}-\tilde{b}_{L}\right.$ mixing) for $\tan \beta=2,10$ and 30 with $m_{\text {top }}=150 \mathrm{GeV}$. The parameters are same as those in Fig. 22.

FIG. 2 Histograms of the mixing matrix element $\left(\tilde{U}_{D}^{\prime}\right)_{1}^{2}\left(s_{L}-\tilde{d}_{L}\right.$ mixing) for $\tan \beta=2,10$ and 30 with $m_{\text {top }}=150 \mathrm{GeV}$. The parameters are same as those in Fig. 2a.

FIG. 2f Histograms of the mixing matrix element $\left(\tilde{U}_{D}^{\prime}\right)_{3}^{2}\left(s_{L}-\tilde{b}_{L}\right.$ mixing) for $\tan \beta=$ 2, 10 and 30 with $m_{\text {top }}=150 \mathrm{GeV}$. The parameters are same as those in Fig. 2a.

FIG. 3 Nucleon decay partial lifetimes for $m_{\text {top }}=150 \mathrm{GeV}$ and $\tan \beta=2$ with $10 \mathrm{GeV} \leq m_{0} \leq 10 \mathrm{TeV}, 10 \mathrm{GeV} \leq M_{g X} \leq 10 \mathrm{TeV}$ and $-5 \leq A_{X} \leq+5$. The lepton in each mode has a lepton number -1 ( " $\nu$ ", "e" and " $\mu$ " mean $\bar{\nu}$, $e^{+}$and $\mu^{+}$, respectively). " $K$ " means a $K$ meson with a $\bar{s}$ quark $\left(K^{+}\right.$or $\left.K^{0}\right)$. $\bar{\nu}$ without a suffix means the total of three neutrinos. The shaded region is excluded experimentally. If the data points with $\tau\left(n \rightarrow K^{0} \bar{\nu}\right)<0.86 \times 10^{32}$ yrs are omitted, the minimum value of each mode is raised to the vertical line. 
FIG. 4a The ratios of partial lifetimes calculated only the wino dressing diagrams $(\tau$ (wino $))$ and those calculated with both wino and gluino dressing diagrams $(\tau$ (total) $)$ for $m_{\text {top }}=150 \mathrm{GeV}$ and $\tan \beta=2$ with $10 \mathrm{GeV} \leq m_{0} \leq 10 \mathrm{TeV}$, $10 \mathrm{GeV} \leq M_{g X} \leq 10 \mathrm{TeV}$ and $-5 \leq A_{X} \leq+5$.

FIG. $4 \mathrm{~b}$ The ratios of partial lifetimes calculated only the wino dressing diagrams and those calculated with both wino and gluino dressing diagrams for $m_{\mathrm{top}}=$ $150 \mathrm{GeV}$ and $\tan \beta=10$ with $10 \mathrm{GeV} \leq m_{0} \leq 10 \mathrm{TeV}, 10 \mathrm{GeV} \leq M_{g X} \leq$ $10 \mathrm{TeV}$ and $-5 \leq A_{X} \leq+5$.

FIG. 4 The ratios of partial lifetimes calculated only the wino dressing diagrams and those calculated with both wino and gluino dressing diagrams for $m_{\text {top }}=$ $150 \mathrm{GeV}$ and $\tan \beta=30$ with $10 \mathrm{GeV} \leq m_{0} \leq 10 \mathrm{TeV}, 10 \mathrm{GeV} \leq M_{g X} \leq$ $10 \mathrm{TeV}$ and $-5 \leq A_{X} \leq+5$.

FIG. 5 A scatter plot of the $p \rightarrow K^{+} \bar{\nu}$ mode lifetime versus the wino-total ratio of the $p \rightarrow K^{0} e^{+}$mode for $m_{\text {top }}=150 \mathrm{GeV}$ and $\tan \beta=10$ with $10 \mathrm{GeV} \leq$ $m_{0} \leq 10 \mathrm{TeV}, 10 \mathrm{GeV} \leq M_{g X} \leq 10 \mathrm{TeV}$ and $-5 \leq A_{X} \leq+5$. The shaded region is excluded experimentally.

FIG. 6a Scatter plots in $\mu-M_{2}$ plane for $m_{\text {top }}=150 \mathrm{GeV}$ and $\tan \beta=2$ with $10 \mathrm{GeV} \leq m_{0} \leq 10 \mathrm{TeV}, 10 \mathrm{GeV} \leq M_{g X} \leq 10 \mathrm{TeV}$ and $-5 \leq A_{X} \leq+5$. Region A is excluded by LEP experiment. Region $\mathrm{B}$ has no radiative breaking solutions (see text). The region plotted with small dots should be excluded by the nucleon decay experiments.

FIG. 6b Scatter plots in $m_{\tilde{d}_{L}}-M_{2}$ plane for $m_{\text {top }}=150 \mathrm{GeV}$ and $\tan \beta=2$ with $10 \mathrm{GeV} \leq m_{0} \leq 10 \mathrm{TeV}, 10 \mathrm{GeV} \leq M_{g X} \leq 10 \mathrm{TeV}$ and $-5 \leq A_{X} \leq+5$. The region plotted with small dots should be excluded by the nucleon decay experiments. 


\section{Figures}

FIG. 1: Examples of dressing diagrams which contribute to the proton decay process $p \rightarrow K^{+} \bar{\nu}$. 
FIG. 2a: Histograms of the mixing matrix element $\left(\tilde{U}_{U}\right)_{2}^{1}\left(u_{L}-\tilde{c}_{L}\right.$ mixing $)$ for $\tan \beta=2,10$ and 30 with $m_{\text {top }}=150 \mathrm{GeV}, 10 \mathrm{GeV} \leq m_{0} \leq 10 \mathrm{TeV}, 10 \mathrm{GeV} \leq$ $M_{g X} \leq 10 \mathrm{TeV}$ and $-5 \leq A_{X} \leq+5$.

FIG. 2b: Histograms of the mixing matrix element $\left(\tilde{U}_{U}\right)_{3}^{1}\left(u_{L}-\tilde{t}_{L}\right.$ mixing) for $\tan \beta=2,10$ and 30 with $m_{\text {top }}=150 \mathrm{GeV}$. The parameters are same as those in Fig. 2a.

FIG. 2c: Histograms of the mixing matrix element $\left(\tilde{U}_{D}^{\prime}\right)_{2}^{1}\left(d_{L}-\tilde{s}_{L}\right.$ mixing) for $\tan \beta=2,10$ and 30 with $m_{\text {top }}=150 \mathrm{GeV}$. The parameters are same as those in Fig. 2a.

FIG. 2d: Histograms of the mixing matrix element $\left(\tilde{U}_{D}^{\prime}\right)_{3}^{1}\left(d_{L}-\tilde{b}_{L}\right.$ mixing) for $\tan \beta=2,10$ and 30 with $m_{\text {top }}=150 \mathrm{GeV}$. The parameters are same as those in Fig. 2a.

FIG. 2e: Histograms of the mixing matrix element $\left(\tilde{U}_{D}^{\prime}\right)_{1}^{2}\left(s_{L}-\tilde{d}_{L}\right.$ mixing) for $\tan \beta=2,10$ and 30 with $m_{\text {top }}=150 \mathrm{GeV}$. The parameters are same as those in Fig. 2a.

FIG. 2f: Histograms of the mixing matrix element $\left(\tilde{U}_{D}^{\prime}\right)_{3}^{2}\left(s_{L}-\tilde{b}_{L}\right.$ mixing $)$ for $\tan \beta=$ 2, 10 and 30 with $m_{\text {top }}=150 \mathrm{GeV}$. The parameters are same as those in Fig. 2a. 
FIG. 3: Nucleon decay partial lifetimes for $m_{\text {top }}=150 \mathrm{GeV}$ and $\tan \beta=2$ with $10 \mathrm{GeV} \leq m_{0} \leq 10 \mathrm{TeV}, 10 \mathrm{GeV} \leq M_{g X} \leq 10 \mathrm{TeV}$ and $-5 \leq A_{X} \leq+5$. The lepton in each mode has a lepton number -1 (" $\nu$ ", " $e$ " and " $\mu$ " mean $\bar{\nu}, e^{+}$and $\mu^{+}$, respectively). " $K$ " means a $K$ meson with a $\bar{s}$ quark $\left(K^{+}\right.$or $\left.K^{0}\right)$. $\bar{\nu}$ without a suffix means the total of three neutrinos. The shaded region is excluded experimentally. If the data points with $\tau\left(n \rightarrow K^{0} \bar{\nu}\right)<0.86 \times 10^{32}$ yrs are omitted, the minimum value of each mode is raised to the vertical line.

FIG. 4a: The ratios of partial lifetimes calculated only the wino dressing diagrams $(\tau$ (wino $))$ and those calculated with both wino and gluino dressing diagrams $(\tau$ (total) $)$ for $m_{\text {top }}=150 \mathrm{GeV}$ and $\tan \beta=2$ with $10 \mathrm{GeV} \leq m_{0} \leq 10 \mathrm{TeV}$, $10 \mathrm{GeV} \leq M_{g X} \leq 10 \mathrm{TeV}$ and $-5 \leq A_{X} \leq+5$.

FIG. 4b: The ratios of partial lifetimes calculated only the wino dressing diagrams and those calculated with both wino and gluino dressing diagrams for $m_{\text {top }}=150$ $\mathrm{GeV}$ and $\tan \beta=10$ with $10 \mathrm{GeV} \leq m_{0} \leq 10 \mathrm{TeV}, 10 \mathrm{GeV} \leq M_{g X} \leq 10 \mathrm{TeV}$ and $-5 \leq A_{X} \leq+5$.

FIG. 4c: The ratios of partial lifetimes calculated only the wino dressing diagrams and those calculated with both wino and gluino dressing diagrams for $m_{\text {top }}=150$ $\mathrm{GeV}$ and $\tan \beta=30$ with $10 \mathrm{GeV} \leq m_{0} \leq 10 \mathrm{TeV}, 10 \mathrm{GeV} \leq M_{g X} \leq 10 \mathrm{TeV}$ and $-5 \leq A_{X} \leq+5$.

FIG. 5: A scatter plot of the $p \rightarrow K^{+} \bar{\nu}$ mode lifetime versus the wino-total ratio of the $p \rightarrow K^{0} e^{+}$mode for $m_{\text {top }}=150 \mathrm{GeV}$ and $\tan \beta=10$ with $10 \mathrm{GeV} \leq m_{0} \leq$ $10 \mathrm{TeV}, 10 \mathrm{GeV} \leq M_{g X} \leq 10 \mathrm{TeV}$ and $-5 \leq A_{X} \leq+5$. The shaded region is excluded experimentally. 
FIG. 6a: Scatter plots in $\mu-M_{2}$ plane for $m_{\mathrm{top}}=150 \mathrm{GeV}$ and $\tan \beta=2$ with $10 \mathrm{GeV} \leq m_{0} \leq 10 \mathrm{TeV}, 10 \mathrm{GeV} \leq M_{g X} \leq 10 \mathrm{TeV}$ and $-5 \leq A_{X} \leq+5$. Region $\mathrm{A}$ is excluded by LEP experiment. Region $\mathrm{B}$ has no radiative breaking solutions (see text). The region plotted with small dots should be excluded by the nucleon decay experiments.

FIG. 6b: Scatter plots in $m_{\tilde{d}_{L}}-M_{2}$ plane for $m_{\text {top }}=150 \mathrm{GeV}$ and $\tan \beta=2$ with $10 \mathrm{GeV} \leq m_{0} \leq 10 \mathrm{TeV}, 10 \mathrm{GeV} \leq M_{g X} \leq 10 \mathrm{TeV}$ and $-5 \leq A_{X} \leq+5$. The region plotted with small dots should be excluded by the nucleon decay experiments. 
This figure "fig1-1.png" is available in "png" format from: http://arxiv.org/ps/hep-ph/9404349v1 
This figure "fig2-1.png" is available in "png" format from: http://arxiv.org/ps/hep-ph/9404349v1 
This figure "fig3-1.png" is available in "png" format from: http://arxiv.org/ps/hep-ph/9404349v1 
This figure "fig4-1.png" is available in "png" format from: http://arxiv.org/ps/hep-ph/9404349v1 
This figure "fig1-2.png" is available in "png" format from: http://arxiv.org/ps/hep-ph/9404349v1 
This figure "fig2-2.png" is available in "png" format from: http://arxiv.org/ps/hep-ph/9404349v1 
This figure "fig3-2.png" is available in "png" format from: http://arxiv.org/ps/hep-ph/9404349v1 
This figure "fig4-2.png" is available in "png" format from: http://arxiv.org/ps/hep-ph/9404349v1 
This figure "fig1-3.png" is available in "png" format from: http://arxiv.org/ps/hep-ph/9404349v1 
This figure "fig2-3.png" is available in "png" format from: http://arxiv.org/ps/hep-ph/9404349v1 
This figure "fig3-3.png" is available in "png" format from: http://arxiv.org/ps/hep-ph/9404349v1 
This figure "fig4-3.png" is available in "png" format from: http://arxiv.org/ps/hep-ph/9404349v1 
This figure "fig2-4.png" is available in "png" format from: http://arxiv.org/ps/hep-ph/9404349v1 
This figure "fig4-4.png" is available in "png" format from: http://arxiv.org/ps/hep-ph/9404349v1 